\title{
Cognitive behavioural therapy improved benzodiazepine discontinuation in chronic insomnia
}

Baillargeon L, Landreville $\mathrm{P}$, Verreault $\mathrm{R}$, et al. Discontinuation of benzodiazepines among older insomniac adults treated with cognitive-behavioural therapy combined with gradual tapering: a randomized trial. CMAJ 2003;169:1015-20.

In older patients with chronic insomnia who have been taking a benzodiazepine nightly for $\geqslant 3$ months, is cognitive
behavioural therapy plus tapering (CBTT) more effective than tapering alone (TA) for inducing benzodiazepine discontinuation?

METHODS

$-1$

Design: randomised controlled trial

Allocation: not concealed.

Blinding: unblinded.

Follow up period: 12 months.

Setting: a university hospital in Sainte-Foy, Québec, Canada.

Patients: 65 patients $\geqslant 50$ years of age (mean age 67 y, 58\% women) with chronic insomnia ( $\geqslant 6$ months); impaired daytime functioning or mood disturbances; nightly benzodiazepine use for $\geqslant 3$ months; and inability to refrain from taking a hypnotic for fear of poor sleep or reported $<80 \%$ sleep efficiency (total sleep time/time in bed $\times 100$ ) over a 2 week period. Exclusion criteria included cognitive impairment, severe psychiatric or physical problems, daytime benzodiazepine use, and consumption of $>3$ alcoholic beverages per day.

$\mathbf{R}_{\mathbf{X}}$

Interventions: 8 weeks of CBTT $(n=35)$ or TA $(n=30)$. Tapering in both groups was supervised by a physician who met weekly with each patient and aimed for dosage reduction of benzodiazepines by $25 \%$ at 1 or 2 week intervals. Cognitive behavioural therapy comprised 8 weekly small group sessions of 90 minutes provided by a psychologist and an optional booster session at 1 month post-treatment; the aim was to reinforce the bed as a cue for sleep, regularise sleep rhythm, enhance sleep efficiency, deal with thoughts that could exacerbate the sleep disorder, and educate about sleep and benzodiazepines.

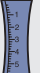

Outcome: benzodiazepine discontinuation, confirmed by blood screening assessed immediately after completion of treatment and at 3 and 12 months.

Patient follow up: $95 \%$

\section{MAIN RESULTS}

Analysis was by intention to treat. More patients in CBTT group than in the TA group reported complete benzodiazepine discontinuation throughout follow up (table).

\section{CONCLUSION}

In older patients with chronic insomnia who had been taking a benzodiazepine every night for $\geqslant 3$ months, cognitive behavioural therapy plus tapering was more effective than tapering alone for inducing benzodiazepine discontinuation.

A modified version of this abstract appears in ACP Journal Club.

\section{Commentary}

$\Delta$ Ithough other studies have explored the effects of CBTT in benzodiazepine withdrawal ${ }^{1}$, the study by Baillargeon et al is important because it is one of few studies that have targeted patients with chronic insomnia.

The findings support the conclusion that CBTT is superior to standard TA for discontinuation of benzodiazepines in older patients with chronic insomnia and that this benefit is sustained over time. The sample is representative of the chronicity of hypnotic use seen in clinical settings. The unblinded design is not ideal, but this weakness is somewhat offset by the large effect observed, statistical adjustment for baseline differences, audiotape monitoring of consultations, and blood screening to confirm self reported benzodiazepine use. Despite the collection of sleep diaries, the effect of treatment on sleep was not reported even though this is an important factor in the assessment of the effectiveness of hypnotic withdrawal regimens. The study findings are relevant to nurses working with older patients with chronic insomnia who take benzodiazepines, although they are not generalisable to poorly motivated and mentally or physically impaired older adults.

Although promising, it is premature to recommend widespread adoption of intensive CBTT for hypnotic withdrawal without first comparing it with less costly strategies or a placebo control condition. For example, in a study of general practice patients, $21 \%$ reported stopping chronic benzodiazepine use after receiving only a letter advising its discontinuation. ${ }^{1}$ Furthermore, regimens that first replace benzodiazepines with non-benzodiazepine hypnotics (eg, zopiclone) have received some attention, although research support is scant. These new hypnotics are easily substituted and have few withdrawal and tolerance effects. ${ }^{2}$ The most cost-effective of these withdrawal regimens still needs to be determined.

Diana E McMillan, RN, PhD Faculty of Nursing, University of Manitoba Winnipeg, Manitoba, Canada

1 Voshaar RC, Gorgels WJ, Mol AJ, et al. Tapering off long-term benzodiazepine use with or without group cognitive-behavioural therapy: three-condition, randomised controlled trial. Br J Psychiatry 2003; 182:498-504.

2 Shapiro CM, Sherman D, Peck DF. Withdrawal from benzodiazepines by initially switching to zopiclone. Eur Psychiatry 1995;10:145s-51s.

Source of funding: National Health Research and Development Program, Health Canada.

Cognitive behavioural therapy plus tapering (СВTT) $v$ tapering alone (TA) in chronic insomnia*

\begin{tabular}{llllr}
\hline Outcome & Follow up & CBTT & TA & RBI (95\% CI) \\
\hline Discontinuation of & Immediately after treatment & $76 \%$ & $38 \%$ & $101 \%(28$ to 246$)$ \\
benzodiazepine & 3 months & $67 \%$ & $34 \%$ & $93 \%(15$ to 248$)$ \\
& 12 months & $70 \%$ & $24 \%$ & $189 \%$ (55 to 487$)$ \\
\hline
\end{tabular}

*Abbreviations defined in glossary; $\mathrm{RBI}, \mathrm{NNT}$, and $\mathrm{Cl}$ calculated from data in article. 